\title{
Polarized alkali vapor with minute-long transverse spin-relaxation time
}

\author{
M. V. Balabas, ${ }^{1}$ T. Karaulanov, ${ }^{2}$ M. P. Ledbetter, ${ }^{2, \text { f }}$ and D. Budker ${ }^{2,3}$ \\ ${ }^{1}$ S. I. Vavilov State Optical Institute, St. Petersburg, 199034 Russia \\ ${ }^{2}$ Department of Physics, University of California at Berkeley, Berkeley, California 94720-7300 \\ ${ }^{3}$ Nuclear Science Division, Lawrence Berkeley National Laboratory, Berkeley CA 94720
}

(Dated: October 24, 2018)

\begin{abstract}
We demonstrate lifetimes of atomic populations and coherences in excess of 60 seconds in alkali vapor cells with inner walls coated with an alkene material. This represents two orders of magnitude improvement over the best paraffin coatings. Such anti-relaxation properties will likely lead to substantial improvements in atomic clocks, magnetometers, quantum memory, and enable sensitive studies of collisional effects and precision measurements of fundamental symmetries.

PACS numbers: PACS. 07.55.Ge, 32.80.Xx, 42.65.-k
\end{abstract}

Long-lived ground-state coherences in atomic vapor cells form the basis for atomic clocks [1], magnetometers [4, 5], quantum memory [6], spin-squeezing and quantum non-demolition measurements [7, 8, and precision measurements of fundamental symmetries [9]. One method for achieving long coherence times is to coat the walls of a cell with an anti-relaxation film such as paraffin [10, 11] or octadecyltrichlorosilane [12. Conventional paraffin coatings are formed from long-chain alkane molecules, supporting approximately $10^{4}$ atom-wall collisions before depolarizing the alkali spins. In this Letter we report on the remarkable anti-relaxation properties of a new, alkene based, coating. With proper experimental arrangements, we realize coherence lifetimes on the order of 1 minute in a $3 \mathrm{~cm}$ diameter cell, corresponding to about $10^{6}$ polarization preserving bounces. To the best of our knowledge, this corresponds to the narrowest electron paramagnetic resonance ever observed.

One of the key ingredients to realizing such long lifetimes is to work in magnetic fields such that the Larmor precession frequency is small compared to the spinexchange rate, and to optically pump the alkali vapor with circularly polarized light. This largely eliminates relaxation due to spin-exchange collisions, the so called spin-exchange relaxation-free (SERF) regime [13, 14. SERF magnetometers presently hold the record for magnetic field sensitivity of any device [15, 16, but usually require operation at temperatures in excess of $150^{\circ} \mathrm{C}$. The alkene coating described here enables operation of such a magnetometer in a room temperature environment, dramatically expanding its useful range of application, especially where low power consumption is important. We present an experimental and theoretical investigation of a room temperature atomic magnetometer operating in the SERF regime. Experiment and theory are in good agreement with each other.

Exchange of atoms between the bulb of the cell and the stem with the $\mathrm{Rb}$ reservoir (Fig. 1) can also produce rapid relaxation and must be mitigated. This can be

*Electronic address: ledbetter@berkeley.edu accomplished by employing a "lockable stem" 17 which provides a coated barrier to reduce the rate of exchange between the bulb and the stem. Finally, gradients of the magnetic field are another source of relaxation, so care must be taken to minimize them.

In Ref. 18 it was reported that cells coated with 1-nonadecene $\left(\mathrm{CH}_{2}-\mathrm{CH}\left(\mathrm{CH}_{2}\right)_{16}-\mathrm{CH}_{2}\right)$ yielded polarization lifetimes of about three seconds. To investigate the alkene-based coating carefully, we prepared three $\mathrm{Rb}$ vapor cells with lockable stems. Cells $\mathrm{C} 1$ and $\mathrm{C} 2 \mathrm{had}$ natural-abundance $\mathrm{Rb}$ and non-ideal locks, cell $\mathrm{C} 3 \mathrm{had}$ ${ }^{87} \mathrm{Rb}$ and a "precision ground" lock. The initial material for the coating preparation was Alpha Olefin Fraction C20-24 from Chevron Phillips (CAS Number 93924-108). A light fraction of the material was removed through vacuum distillation at $80^{\circ} \mathrm{C}$. The remains were used as the coating material. Coatings were prepared with the procedure described in Refs. [4, 19, except the temperature was $175^{\circ} \mathrm{C}$ instead of $220^{\circ} \mathrm{C}$. After preparation, the cells were cured at $70^{\circ} \mathrm{C}$ for several hours. Cell $\mathrm{C} 1$ had a polarization lifetime of 60 seconds, while cells $\mathrm{C} 2$ and C3 had polarization lifetimes of about 15 seconds. The measurements presented here were obtained with cell $\mathrm{C} 1$.

The experimental setup is shown in Fig. 1(a). The coated cell was placed inside four layers of mu-metal and one layer of ferrite [16] shielding. A circularly polarized pump beam, propagating in the $z$ direction, tuned near the $F=2 \rightarrow F^{\prime}$ D1 transitions of ${ }^{85} \mathrm{Rb}$, optically pumped the alkali spins. Spin precession was monitored via optical rotation of linearly polarized probe light, propagating in the $x$ direction, tuned about $1.5 \mathrm{GHz}$ to the blue of the $F=3 \rightarrow F^{\prime}$ D1 transitions of ${ }^{85} \mathrm{Rb}$. Optical rotation, scaling roughly as the inverse of detuning, was dominated by ${ }^{85} \mathrm{Rb}$, however there was some contribution from ${ }^{87} \mathrm{Rb}$. Typical probe power was $\approx 2 \mu \mathrm{W}$, although much higher probe power could be used without incurring substantial additional broadening since the probe was tuned far off resonance. Pump power ranged from $0-2 \mu \mathrm{W}$. Most of the measurements were performed at a temperature of $30^{\circ} \mathrm{C}$ where the $\mathrm{Rb}$ vapor density was $n \approx 1.5 \times 10^{10} \mathrm{~cm}^{-3}$, measured by transmission of a weak probe beam. The orientation of the cell could be manipulated from outside the magnetic shields so that 


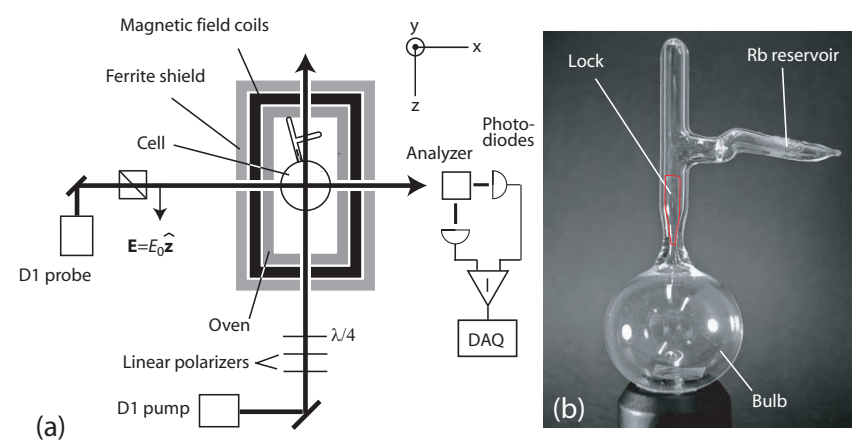

FIG. 1: Experimental setup: (a) Four mu-metal shields (not shown) surround the ferrite shield. The ferrite shield is a cylinder, $15.2 \mathrm{~cm}$ long and $11.4 \mathrm{~cm}$ in diameter (inner dimensions) with $1.3 \mathrm{~cm}$ thick walls. A set of coils provides control over the magnetic fields. An oven, heated by a twisted pair of 30 gauge copper wire and $\mathrm{AC}$ current, provided temperature control. (b) Photo of the coated cell.

the lock could be opened and closed without opening the shields. With the lock open, polarization lifetimes were much shorter than with the lock closed, approximately 3 seconds. Geometry dictated that the stem and locking bullet were nearly horizontal, producing a lock of variable quality: the longitudinal relaxation time varied by as much as a factor of four from run-to-run.

Using the apparatus shown in Fig. 1(a) we investigated the relaxation of both the longitudinal and transverse (with respect to magnetic field) components of spin polarization. Longitudinal relaxation was measured by first applying a magnetic field parallel to the pump beam, and then adiabatically rotating the magnetic field into the direction of the probe beam, and subsequently monitoring optical rotation of the probe as the longitudinal polarization decayed. To investigate transverse relaxation, we observed the transient response of the alkali spins to a non-adiabatic change in the magnetic field, either by (1) pumping the spins in zero magnetic field and applying a step in $B_{y}$, or (2) by pumping the spins in a finite bias magnetic field $B_{z}$ and then applying a short pulse of magnetic field $B_{x}$, similar to RF excitation pulses in nuclear magnetic resonance. We also made high field (10-20 G) measurements of the longitudinal relaxation time using an apparatus similar to that in Ref. [20].

Figure 2 shows optical rotation of the probe due to longitudinal (a) and transverse (b) polarization when the cell was performing optimally. The decay of longitudinal polarization is well described by two exponentials with fast and slow time constants $T_{1 f}=8 \mathrm{~s}$ and $T_{1 s}=53 \mathrm{~s}$, respectively. Such biexponential decays arise from several competing processes of electron spin-destruction collisions with the cell walls, residual relaxation due to collisions with the reservoir, and alkali-alkali spin-exchange collisions 20].

Figure 2(b) shows the transient response to a step in the magnetic field $B_{y} \approx 0.2 \mu \mathrm{G}$ after pumping at zero
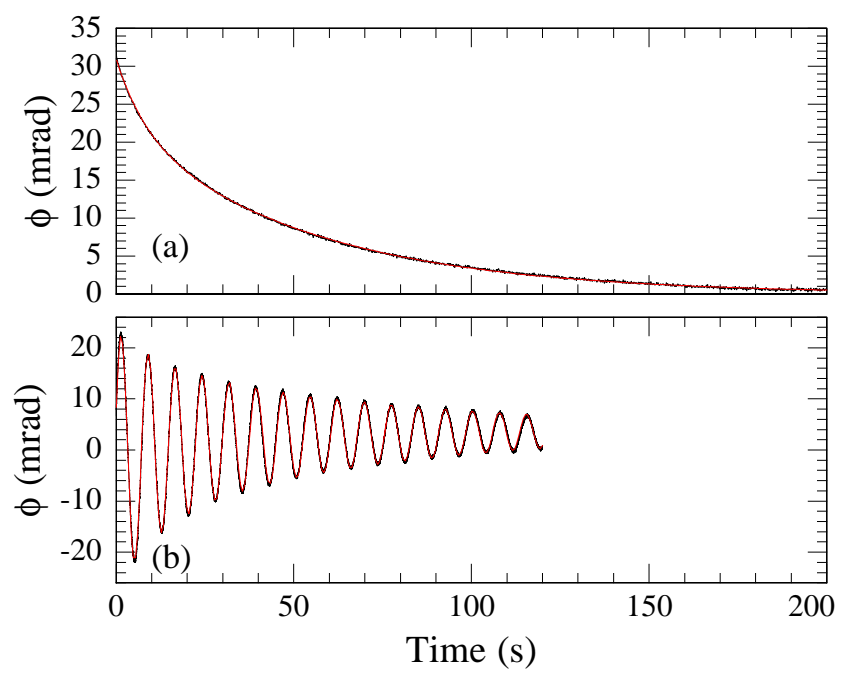

FIG. 2: (color online) (a) Decay of longitudinal polarization. The red trace overlaying the data is a fit to two decaying exponentials, with fast and slow decay times of 8 and 53 seconds, respectively. (b) Response of alkali spin polarization to a transient of the magnetic field. The fast and slow decay times are 13 and 77 seconds.

magnetic field. In such low magnetic fields, the transient response is well described (as indicated by the fitted curve overlaying the data) by an oscillating signal with a single frequency and a return to steady state, with fast and slow decays characterized by lifetimes $T_{2 f}$ and $T_{2 s}$. For the data shown in Fig. 2(b), $T_{2 f}=13 \mathrm{~s}$ and $T_{2 s}=77 \mathrm{~s}$. The presence of only a single frequency oscillation is because the two isotopes "lock" together in the SERF regime in larger magnetic fields we see the appearance of two frequencies corresponding to free precession of either isotope in the absence of spin-exchange collisions. We note that the low-field measurements shown in Fig. 2 were not repeatable: subsequent values of both $T_{1 s}$ and $T_{2 s}$ were somewhat shorter, around 25-35 s. Under these conditions, the fast relaxation was not apparent, and curves were fit with a single decay time, $T_{1 s}$ or $T_{2 s}$. High field measurements of the polarization lifetime, with the stem oriented vertically, were consistently long over the course of several months, in the range of 40-50 seconds. Hence, we suspect that minute-long lifetimes in low field were difficult to reproduce because of the variable quality of the locking stem in the nearly horizontal position.

The temperature dependences of the density and longitudinal relaxation rate may be useful in characterizing the properties of the coating, and are shown in Fig. 3. In acquiring these data, we let the oven and cell equilibrate for several hours at each temperature before measuring the density and lifetime. The lock was closed the entire time. We see a sharp drop in density and an increase in relaxation rate as we pass through the melting transition of the coating at $\approx 33^{\circ} \mathrm{C}$. This behavior was repeatable and without significant hysteresis, as data points were ac- 


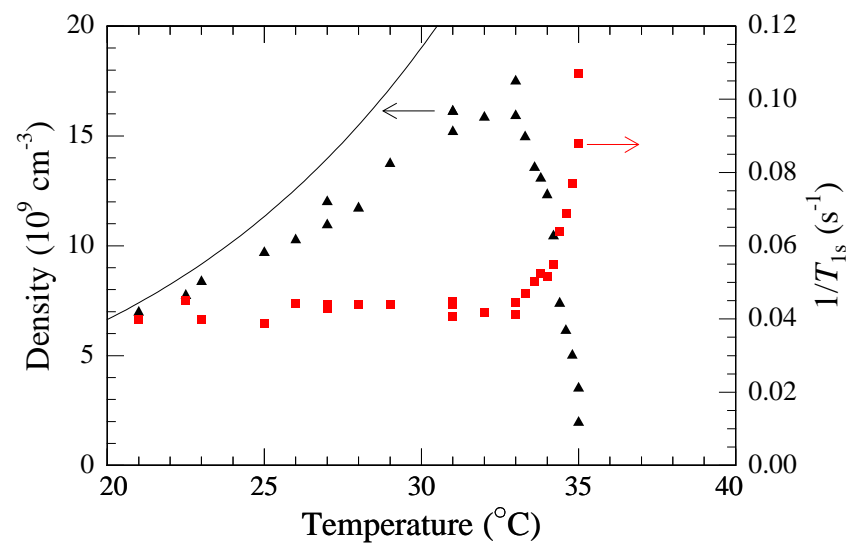

FIG. 3: (color online) Density (triangles) and relaxation rate (squares) as a function of temperature. Density appears to loosely follow that of a saturated vapor (solid curve) until it drops rapidly at $33^{\circ} \mathrm{C}$, accompanied by a significant increase in relaxation rate.

quired non-sequentially. The solid curve represents the expected density for a saturated vapor 21, which begins to deviate from the measured density at relatively low temperature.

We now turn to a discussion of the effects of spin exchange in a low-density vapor in very low magnetic fields. When the Larmor precession frequency is small compared to the spin-exchange rate $1 / T_{e x}=n \sigma_{e x} v$ ( $n$ is the number density, $\sigma_{e x}=1.9 \times 10^{-14} \mathrm{~cm}^{2}$ is the spin-exchange cross section for $\mathrm{Rb}$ [22], and $v$ is the mean relative thermal velocity), spin-exchange collisions produce relaxation that is quadratic in the magnetic field and modify the effective gyromagnetic ratio, both of which depend on the degree of spin polarization [14. Figure 4 shows the magneticfield dependence of the transverse relaxation rate, $1 / T_{2 s}$, for several pump powers. For these data, transverse coherences were produced by applying a short $(0.2 \mathrm{~s})$ pulse of magnetic field in the $x$ direction in the presence of a static field $B_{z}$. The solid curves overlaying the data are fits to $A+A_{S E} B_{z}^{2}$, where $A$ represents relaxation due to wall collisions, pump light, and gradients, and $A_{S E}$ represents the contribution to broadening from spin-exchange collisions. Relaxation deviates from the quadratic behavior shown in Fig. 4 as the magnetic field is increased, reaching an asymptotic level of $1 / T_{2 s} \approx 3 \mathrm{~s}^{-1}$ at $\approx 1 \mathrm{mG}$. At low magnetic field, increasing pump power produces power broadening, however, at higher magnetic fields, high pump power reduces spin-exchange relaxation by preferentially populating the stretched state, which is immune to spin-exchange relaxation 23 .

For a single isotope with a spin-temperature distribution and low polarization, spin-exchange relaxation is given by 24

$$
\Gamma_{S E}=\omega_{0}^{2} T_{e x} \frac{q_{0}^{2}-(2 I+1)^{2}}{2 q_{0}},
$$

where $\omega_{0}=g_{s} \mu_{B} B / q_{0} \hbar, I$ is the nuclear spin, and

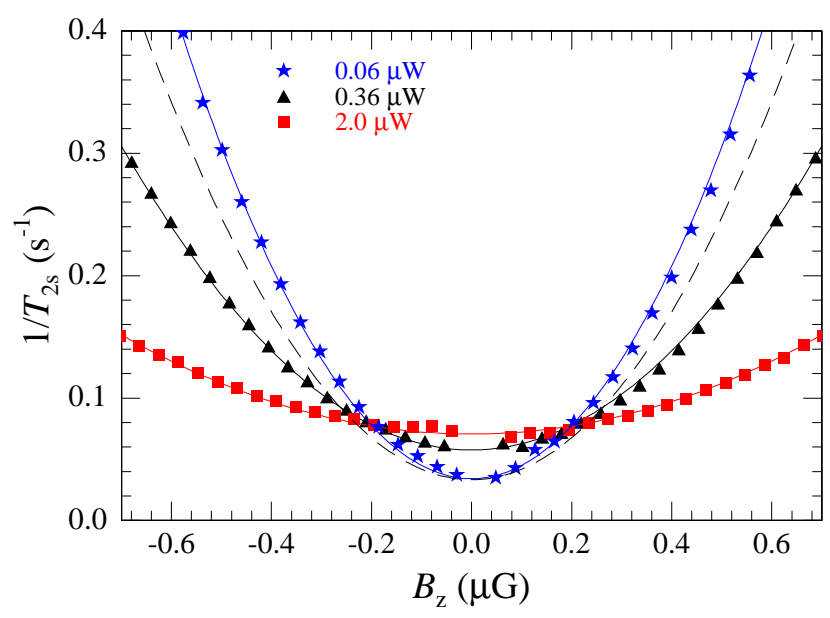

FIG. 4: (color online) Transverse relaxation rate as a function of magnetic field for several values of the pump power. The smooth curves overlaying the data are fits to a curve described in the text. The dashed curve is the relaxation rate in the very low polarization limit given by Eq. 1 for nuclear spin $I=5 / 2$.

$q_{0}=[I(I+1)+S(S+1)] / S(S+1)$ is the nuclear slowingdown factor. The dashed curve in Fig. 4 is the expected relaxation rate in the low polarization limit for a vapor of pure ${ }^{85} \mathrm{Rb}(I=5 / 2)$ given by Eq. (1), with $T_{e x}$ determined by transmission measurements of the density. Equation (1) appears close to accounting for relaxation at low light power, however there is clearly some discrepancy, presumably due to the presence of two isotopes.

The gyromagnetic ratio also varies significantly with pump power. In order to compare with theoretical calculations (see below) it is convenient to plot the measured spin-exchange broadening $A_{S E}$ as a function of the effective gyromagnetic ratio $\gamma$ (Fig. 5. triangles). It is interesting to note that there is a linear relationship between these two parameters, as indicated by the linear fit overlaying the data. It is also worth noting that, in these measurements, spin-exchange broadening approaches an asymptotic value of about $0.2 \mathrm{~s}^{-1} / \mu \mathrm{G}^{2}$ at high power due to the presence of two isotopes, as can be seen by the clustering of data points at high light power, despite the increasing size of light power steps. In an isotopically pure vapor, relaxation due to spin-exchange collisions could be largely eliminated at high pump power by hyperfine pumping, similar to the light narrowing observed in Ref. [23.

To further investigate the effects of spin-exchange collisions, we performed numerical simulations following the approach used in Refs. [25, 26]. The contributions to evolution of the ground state density matrix $\rho_{j}$ for isotope $j$ due to hyperfine splitting, Zeeman splitting, optical pumping, spin-destruction, and spin-exchange are, 


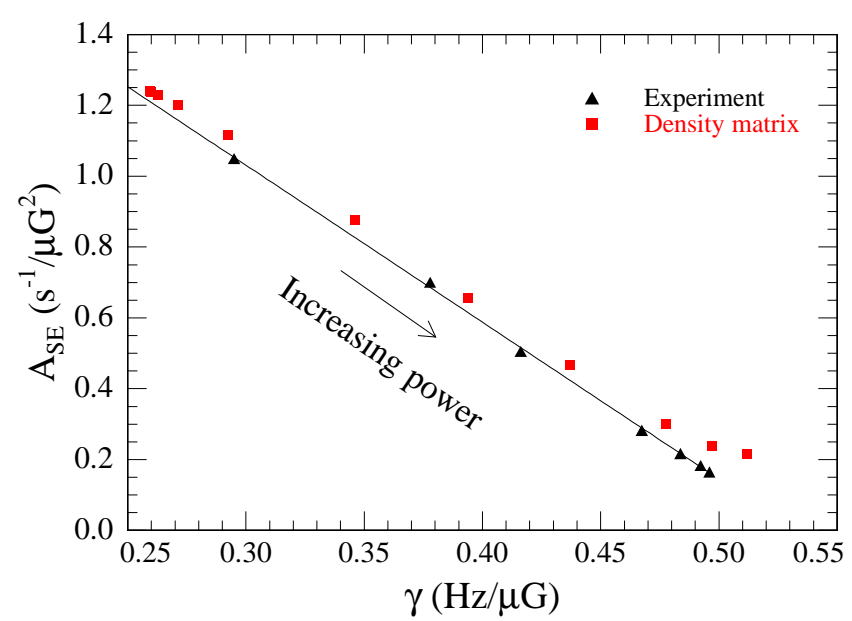

FIG. 5: (color online) Triangles show experimental measurements of spin-exchange broadening $A_{S E}$ vs. the effective gyromagnetic ratio $\gamma$ for pump power ranging from $0.06 \mu \mathrm{W}$ to $2 \mu \mathrm{W}$. The straight line overlaying the data is a linear fit. Squares show the results of density matrix calculations.

respectively:

$$
\begin{aligned}
\frac{d \rho_{j}}{d t} & =\frac{a_{j}}{i \hbar}\left[\mathbf{I}_{j} \cdot \mathbf{S}_{j}, \rho_{j}\right]+\frac{g_{s} \mu_{B}}{i \hbar}\left[\mathbf{B} \cdot \mathbf{S}_{j}, \rho_{j}\right] \\
& +R\left[\phi_{85}\left(1+2 \hat{\mathbf{z}} \cdot \mathbf{S}_{85}\right)-\rho_{j}\right]+\frac{\phi_{j}-\rho_{j}}{T_{s d}} \\
& +\sum_{k} \frac{\phi_{j}\left(1+4\left\langle\mathbf{S}_{k}\right\rangle \cdot \mathbf{S}_{j}\right)-\rho_{j}}{T_{e x, j k}} .
\end{aligned}
$$

Here $a_{j}$ is the hyperfine constant, $\mathbf{I}_{j}$ is the nuclear spin, $g_{s}$ is the Landé factor for the electron, $\mu_{B}$ is the Bohr magneton, $R$ is the optical pumping rate for ${ }^{85} \mathrm{Rb}$ (there is no optical pumping of ${ }^{87} \mathrm{Rb}$ since the pump light is resonant only with ${ }^{85} \mathrm{Rb}$ transitions), and $\phi_{j}=\rho_{j} / 4+\mathbf{S}_{j} \cdot \rho_{j} \mathbf{S}_{j}$ is the purely nuclear part of the density matrix. The spindestruction rate $T_{s d}$ is determined from measurements of $T_{1}$, and the spin-exchange rates $T_{e x, j k}$ are determined by the measured alkali density and the known cross-sections. The transient response to a pulse of magnetic field in the $y$ direction and subsequent precession around a static field in the $z$ direction is determined by numerically integrating Eq. (2), starting from a spin-temperature dis- tribution along the $z$ axis. We extract the $x$ component of electron spin polarization, weighted by isotopic abundance $\eta_{j},\left\langle S_{x}\right\rangle=\eta_{85}\left\langle S_{x, 85}\right\rangle+\eta_{87}\left\langle S_{x, 87}\right\rangle$, a reasonable approximation of the experimental observable, and fit this to a decaying sinusoid. The squares in Fig. 5 show the results of simulations. Experiment and simulation are in good agreement for low light power, although there is some small systematic offset, which we attribute to uncertainty in the alkali vapor density. At higher light power, the simulation deviates from experiment, presumably because the optical pumping term in Eq. (2) is correct in the limit of unresolved hyperfine structure, and therefore cannot account for hyperfine pumping present in the experiment.

In conclusion, we have demonstrated that an alkene coating can support up to $10^{6}$ alkali-wall collisions before depolarizing the alkali spins when all other sources of relaxation are properly mitigated. This represents an improvement by nearly a factor of 100 over traditional coatings. We demonstrate here that cells employing such a coating can enable operation of a SERF magnetometer in a room temperature environment, dramatically expanding the scope of applications for such magnetometers. In addition to magnetometry, anti-relaxation coatings are used in a number of other contexts in both pure and applied research. As we outlined here, alkene coatings can be used to study the effects of spin-exchange collisions in very low density environments, and may be of use for investigating more subtle atom-atom collisions. Alkali vapor cells utilizing such coatings may also dramatically improve the performance of atomic clocks, depending on the nature of the hyperfine shifts associated with atomwall collisions, a subject of future investigation. Alkene coated cells may greatly enhance the lifetime of quantum memory applications [6] or the storage time of light in "slow-light" experiments [28, 29. In the context of geophysical measurements, extremely narrow lines can reduce orientation dependent "heading errors" due to the non-linear Zeeman effect, a significant issue in geomagnetic surveying [27]. While spin-exchange relaxation is difficult to completely eliminate at high field, the use of only a single isotope and hyperfine pumping may reduce such relaxation considerably. This work was supported by the ONR MURI program and by the NSF.
[1] H. G. Robinson, and C. E. Johnson, Applied Physics Letters 40, 771 (1982).

[2] A. Risley, S. Jarvis, and J. Vanier, Journal of Applied Physics 51, 4571 (1980).

[3] C. Rahman, and H. G. Robinson, IEEE Journal of Quantum Electronics 23, 452 (1987).

[4] E. B. Alexandrov, and V. A. Bonch-Bruevich, Optical Engineering 31, 711 (1992).

[5] D. Budker, and M. V. Romalis, Nature Physics 3, 227 (2007).
[6] B. Julsgaard et al., Nature 432, 482 (2004).

[7] A. Kuzmich, L. Mandel, and N. P. Bigelow, Phys. Rev. Lett. 85, 1594 (2000).

[8] W.Wasilewski et al., Phys. Rev. Lett. 104, 133601 (2010).

[9] W.C. Griffith et al., Phys. Rev. Lett. 102 101601, (2009).

[10] H. G. Robinson, E. S. Ensberg, and H. G. Dehmelt, Bull. Am. Phys. Soc. 3, 9 (1958).

[11] M. A. Bouchiat, and J. Brossel, Physical Review 147, 41 (1966). 
[12] S. J. Seltzer, P. J. Meares, and M. V. Romalis, Physical Review A 75, 051407(R) (2007).

[13] J.C. Allred, R.N. Lyman, T.W. Kornack, and M.V. Romalis, Phys. Rev. Lett. 89, 130801 (2002).

[14] W. Happer, and H. Tang, Phys. Rev. Lett. 31, 273 (1973).

[15] I. K. Kominis, T. W. Kornack, J. C. Allred, and M. V. Romalis, Nature 422, 596 (2003).

[16] T. W. Kornack et al., Appl. Phys. Lett. 90, 223501 (2007).

[17] T. Karaulanov et al., Phys. Rev. A 79, 012902 (2009)

[18] M. V. Balabas et al., Optics Express 18, 5825 (2010).

[19] M. V. Balabas, Ph.D. Thesis, (Vavilov State Optical Institute), (1995).

[20] M. T. Graf et al., Phys. Rev. A 72, 023401 (2005).

[21] CRC Handbook of Chemistry and Physics, 74th ed.
(CRC Press, Cleveland, 1993), pp. 4124.

[22] D. K. Walter, W. M. Griffith, and W. Happer, Phys. Rev. Lett. 88, 093004 (2002).

[23] S. Appelt, A. B. Baranga, A. R. Young, W. Happer, Phys. Rev. A 59, 2078 (1999).

[24] W. Happer, and A. C. Tam, Phys. Rev. A 16, 1877 (1977).

[25] S. Appelt et al Phys. Rev. A 58, 1412 (1998).

[26] I. M. Savukov and M. V. Romalis, Phys. Rev. A 71, 023405 (2005).

[27] E. B. Alexandrov, Phys. Scr., T 105, 27 (2003).

[28] D. Budker, D. F. Kimball, S. M. Rochester, and V. V. Yashchuk, Phys. Rev. Lett. 83, 1767 (1999).

[29] M. Klein et al., Journal of Modern Optics 53, 2583 (2006). 